\title{
Aplicação de GPR na avaliação de reservas minerais - o caso do Depósito de Areia Morro Grande, município de Porto Grande, Amapá
}

Welitom Rodrigues Borges (Instituto de Geociências, UnB), Alexandre Lisboa Lago (DIGEOF/ERJ, CPRM), Almir Araújo

Pacheco (SUREG/BE, CPRM), Jorge Armando Freitas do Amaral (SUREG/BE, CPRM), Sheila Gatinho Texeira

(SUREG/BE, CPRM), Ruben Sardou Filho (CPRM, Brasília), Daniel Francisco Pimenta Quintas (SEICOM/AP, CPRM)

Copyright 2019, SBGf - Sociedade Brasileira de Geofísica

This paper was prepared for presentation during the $16^{\text {th }}$ International Congress of the Brazilian Geophysical Society held in Rio de Janeiro, Brazil, 19-22 August 2019.

Contents of this paper were reviewed by the Technical Committee of the $16^{\text {th }}$ International Congress of the Brazilian Geophysical Society and do not necessarily represent any position of the SBGf, its officers or members. Electronic reproduction or storage of any part of this paper for commercial purposes without the written consent of the Brazilian Geophysical Society is prohibited.

\section{Abstract}

The application of geophysical methods in the determination of volumes of sandy deposits minimizes the cost with surveys and allows the more efficient estimation of the mineral reserves. In this work are shown GPR results applied in the research of the thicknesses and volumes of sandy layers in the Morro Branco Sand Deposit, in Amapá. In the field, a GPR coupled to a 250 $\mathrm{MHz}$ shielded antenna was used, and the layer thicknesses were calibrated in the sand outcrops. The results confirm the efficiency of GPR in the determination of layer thicknesses, which allowed the estimation of the volumes of layers of solid sand $\left(280,000 \mathrm{~m}^{3}\right)$ and gravels with sandy matrix $\left(824,000 \mathrm{~m}^{3}\right)$.

\section{Introdução}

O Projeto Materiais de Construção da Região de Macapá, desenvolvido pela GEREMI/SUREG-BE, teve como finalidade a avaliação global dos depósitos de Areia/Cascalho, Argila e Brita, abrangendo os municípios de Porto Grande, Ferreira Gomes, Mazagão, Santana e Macapá, estado do Amapá. Englobou-se nesta avaliação a caracterização tecnológica dos materiais, questões ambientais do processo de extração, definição de reservas e a proposição de novas áreas para a exploração; que, sobretudo, suprem empreendimentos da Construção Civil da cidade de Macapá.

Face ao exposto, a natureza não invasiva dos métodos geofísicos, aliados ao baixo custo operacional, a rapidez e a facilidade de realização do levantamento, torna-os uma excelente ferramenta para o imageamento de depósitos de areia em exploração no Estado do Amapá. Além disso, algumas técnicas geofísicas possibilitam a obtenção de dados contínuos sobre o local investigado, o que reduz a necessidade de um grande volume de dados diretos e específicos (furos de sondagem, trincheiras, etc.), assim como permitem uma análise temporal e espacial mais detalhada dos depósitos. Dentro deste contexto, usou-se o método Ground Penetrating Radar (GPR) com o objetivo de estimar a profundidade e a geometria de um dos depósitos de areia do município de Porto Grande, Amapá.

\section{Metodologia}

O radar de penetração no solo (GPR) é um método eletromagnético que irradia pulsos eletromagnéticos para o meio com altas frequências (20 MHz até $2600 \mathrm{MHz}$ ) e registra as reflexões eletromagnéticas causadas por contraste de impedância elétrica nas estruturas subterrâneas. A propagação da onda depende principalmente do efeito de polarização da onda eletromagnética (permissividade dielétrica) e da atenuação do sinal causado pelo aumento da condutividade elétrica.

O método GPR é amplamente usado em estudos geológicos, e extremamente eficiente para mapear ambientes dielétricos, como as camadas arenosas (BRISTOW e JOL, 2003). Em função de sua eficiência nestes ambientes optou-se pelo o uso do mesmo na estimativa do volume de depósitos arenosos na região norte de Macapá, Amapá.

$\mathrm{Na}$ aquisição de dados de GPR utilizou-se o sistema MALA ProEx (fabricado pela Mala GeoScience), que consiste em um módulo de aquisição conectado a um par de antenas blindadas de $250 \mathrm{MHz}$. Na área do areal Morro Branco realizaram-se 5 seções de GPR em locais onde foi possível passar o equipamento em função da vegetação densa (Figura 1).

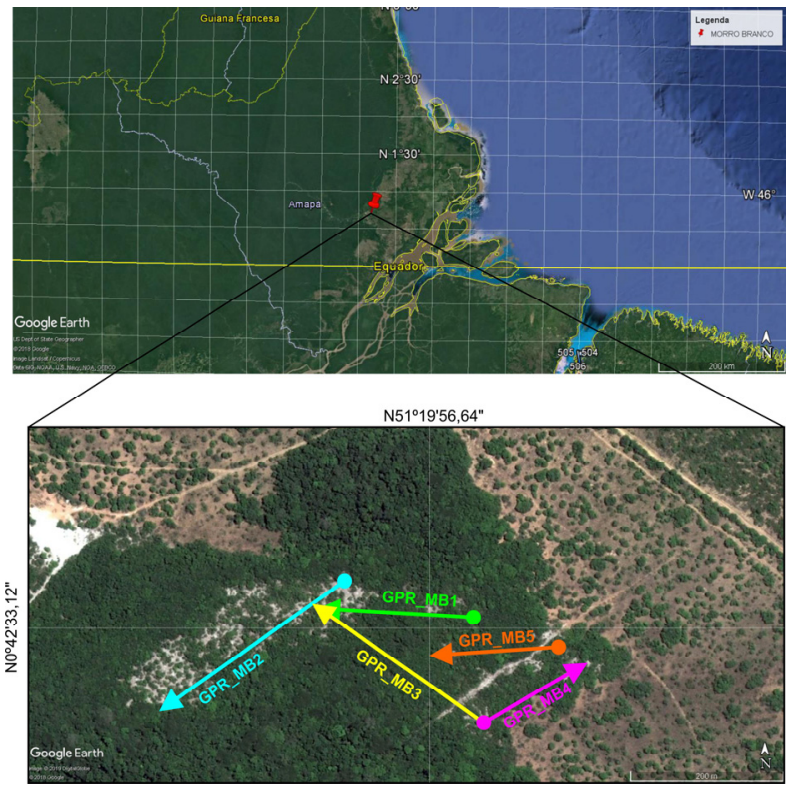

Figura 1 - Imagem aérea da área do areal Morro Branco, com a localização das 5 seções de GPR, município de Porto Grande, Amapá. 
Os dados foram adquiridos deslocando-se o GPR através da técnica do afastamento constante (common offset, Figura 2). Os parâmetros de aquisição de dados foram:

- A frequência de amostragem temporal foi de $1250 \mathrm{MHz}$, o intervalo de amostragem temporal foi de 0,3999 ns, o número de amostras por traço foi de 888 , o que resultou em uma janela temporal de $355 \mathrm{~ns}$;

- O intervalo de amostragem entre os traços foi de $5 \mathrm{~cm}$. Esta amostragem foi realizada automaticamente com o uso de um odômetro eletrônico (roda).
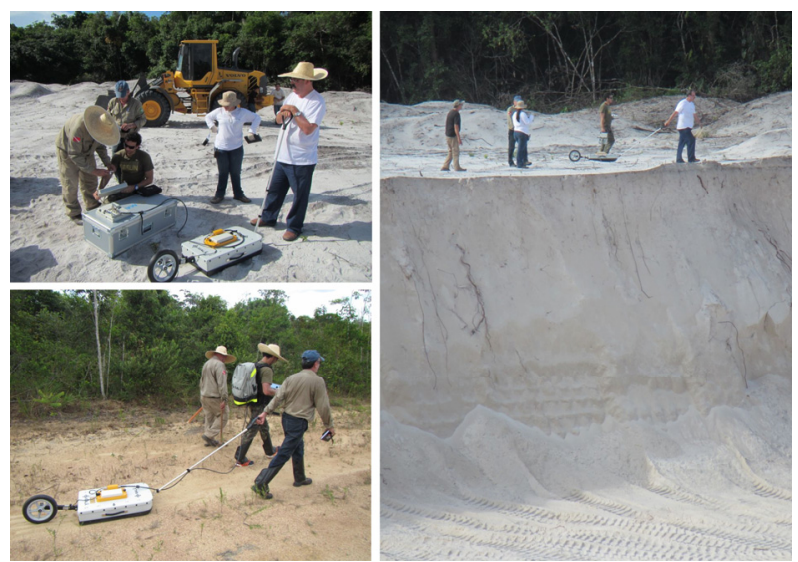

Figura 2 - Fotografias ilustram as aquisições de GPR, com o sistema RAMAC e antenas blindadas de $250 \mathrm{MHz}$, no areal Morro Branco.

\section{Processamento}

O processamento dos dados de campo adquiridos pelo método GPR 2D foram processados no software ReflexW, versão 7.0 (Sandmeier, 2012). A rotina de processamento dos dados $2 \mathrm{D}$ consistiu em:

- $\quad$ IMPORT - conversão de formato dos arquivos ( ${ }^{*}$.RD3 - saída do equipamento MALA ProEX, para o formato *.dat - arquivo do ReflexW);

- SET TIME ZERO (ajuste do tempo zero) ajuste das primeiras chegadas da onda eletromagnética;

- ENERGY DECAY (ganho) - aplicado para recuperação da amplitude do sinal eletromagnético atenuada durante a propagação do sinal no meio;

- BANDPASS (filtro 1D) - eliminação dos ruídos eletrônicos e estáticos inerentes ao sistema;

- DIFFRACTION STACK MIGRATION - processo utilizado para colapsar as hipérboles de difração. A migração é um processo inverso no qual as ondas registradas são propagadas de volta à localização correspondente ao difrator;
- $\quad$ TIMEDEPTH CONVERSION - conversão dos perfis de tempo para profundidade. A velocidade de conversão, utilizada nesta etapa, foi obtida através da medida da profundidade real de alguns refletores nas frentes de lavra de areia.

No areal Morro Branco, onde se executaram 5 perfis de GPR, a velocidade eletromagnética calculada a partir de correlação entre um refletor horizontal identificado no tempo duplo de 56 ns e a profundidade de 4,2 metros medida em campo, foi de $0,15 \mathrm{~m} / \mathrm{ns}$ (Figura 3 ). Esta velocidade foi usada na conversão dos radargramas de tempo duplo para profundidade.

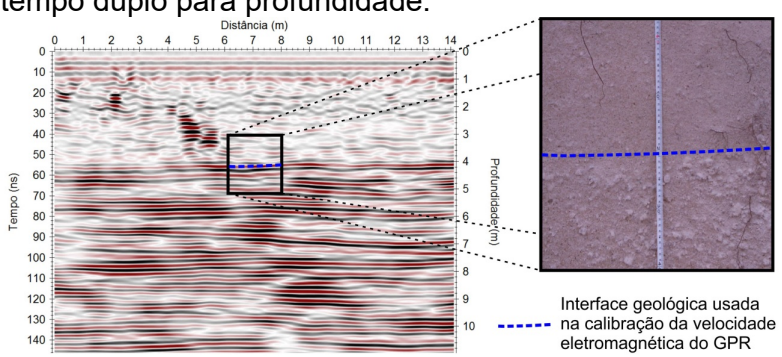

Figura 3 - Seção de GPR, de calibração da velocidade da onda eletromagnética, obtida no areal Morro Branco. Em detalhe a fotografia evidencia a interface entre a areia maciça e o cascalho com matriz arenosa.

\section{Interpretação e Discussão dos Resultados}

A equipe analisou os resultados de GPR através da correlação entre a geometria e a amplitude dos eventos refletores. Nas seções de radar notam-se padrões distintos de reflexão que traduzem o comportamento elétrico do meio à passagem dos campos eletromagnéticos de altas frequências.

A areia branca que atualmente encontra-se em exploração, apresenta o aspecto maciço, com poucos e esparsos clastos de quartzo leitoso. Em função da proximidade com a superfície notam-se inúmeras raízes dentro do pacote de areia maciça. O padrão de reflexão (PR1) característico para este pacote sedimentar é de ausência de refletores contínuos e horizontalizados, uma vez que inexistem interfaces com impedância elétrica capaz que produzir tais refletores. Registram-se, dentro deste padrão de reflexão, inúmeras hipérboles de difração causadas por raízes, alguns blocos de quartzo centimétricos e raros buracos de pequenos animais (Figura 4). 

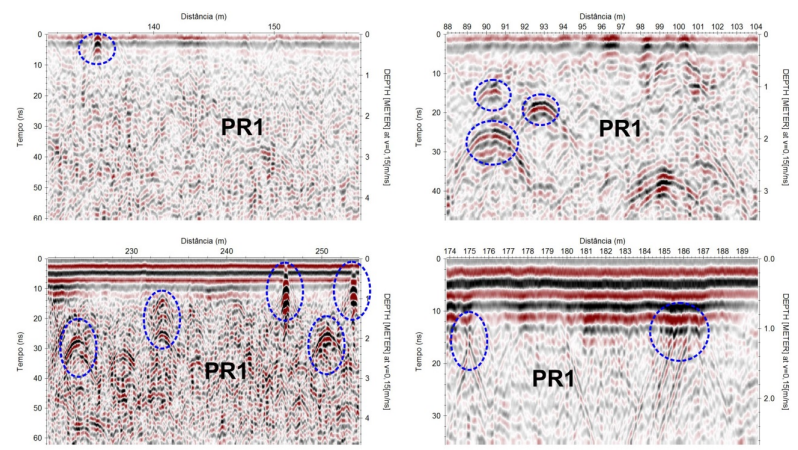

hipérboles de difração causada pela presença de raizes, clastos de

Figura 4 - Seções de GPR evidenciam o padrão de reflexão (PR1) característico do pacote de areia maciça com algumas hipérboles relacionadas a presença de raízes, clastos de quartzo e buracos de animais.

O cascalho com matriz arenosa é pouco utilizado atualmente pelas empresas de extração de areia, em função da necessidade de peneiramento do mesmo. $O$ cascalho ocorre em camadas horizontalizadas e decimétricas que se alternam com camadas centimétricas a decimétricas de areia maciça. Em função da impedância elétrica entre estas camadas, notam-se refletores horizontalizados e de alta amplitude nas profundidades em que estas ocorrem, o que caracteriza o PR3 (Figura 5).
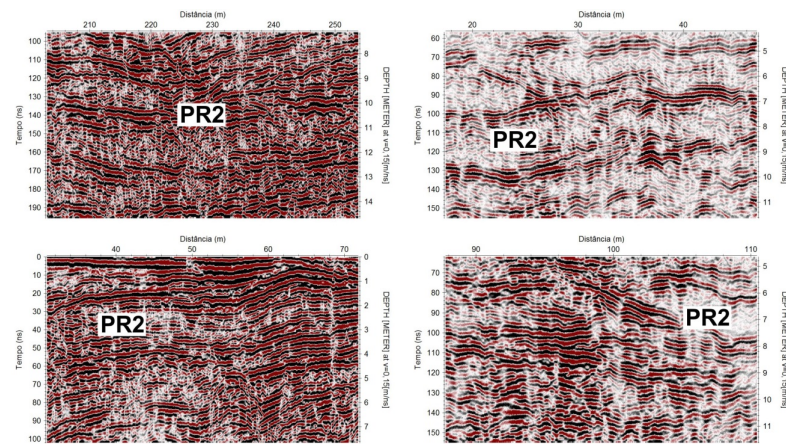

Figura 5 - Seções de GPR evidenciam o padrão de reflexão (PR2) característico do pacote de cascalho com matriz arenosa que se alternam com camadas delgadas de areia maciça.

O terceiro padrão de reflexão (PR3) identificado nas áreas é interpretado como o embasamento rochoso nas áreas, uma vez que possui refletores característicos de superfícies de erosão, e ocorre abaixo dos demais padrões de reflexão (Figura 6). Ele é registrado tanto abaixo do padrão PR2, como do padrão PR1. Em nenhum local do campo registrou-se afloramentos rochosos, deste modo esta interpretação é fundamentada apenas no padrão característico de reflexão do GPR.

As interpretações dos perfis de GPR executados no areal Morro Branco ocorreram no software REFLEXW.
Posteriormente desenvolveu-se a edição das imagens (inserção da legenda e padronização das figuras) no software Corel Draw, com o objetivo de melhorar a resolução das imagens (Figura 7).

Em função do tempo em campo e da elevada quantidade de vegetação na área, não houve uma amostragem regular (malha) de perfis de GPR para a realização de uma estimativa de reserva medida de areia. Deste modo, optou-se por realizar uma análise de reserva inferida, uma vez que a execução dos perfis de GPR foi desenvolvida ao longo de estradas e caminhos próximos às frentes de lavra de areia.

Assim, as interpretações dos dados de GPR, realizadas no software REFLEXW, possibilitaram a elaboração de histogramas de espessura das camadas de areia maciça (Figura 8) e de cascalho com matriz arenosa (Figura 9).

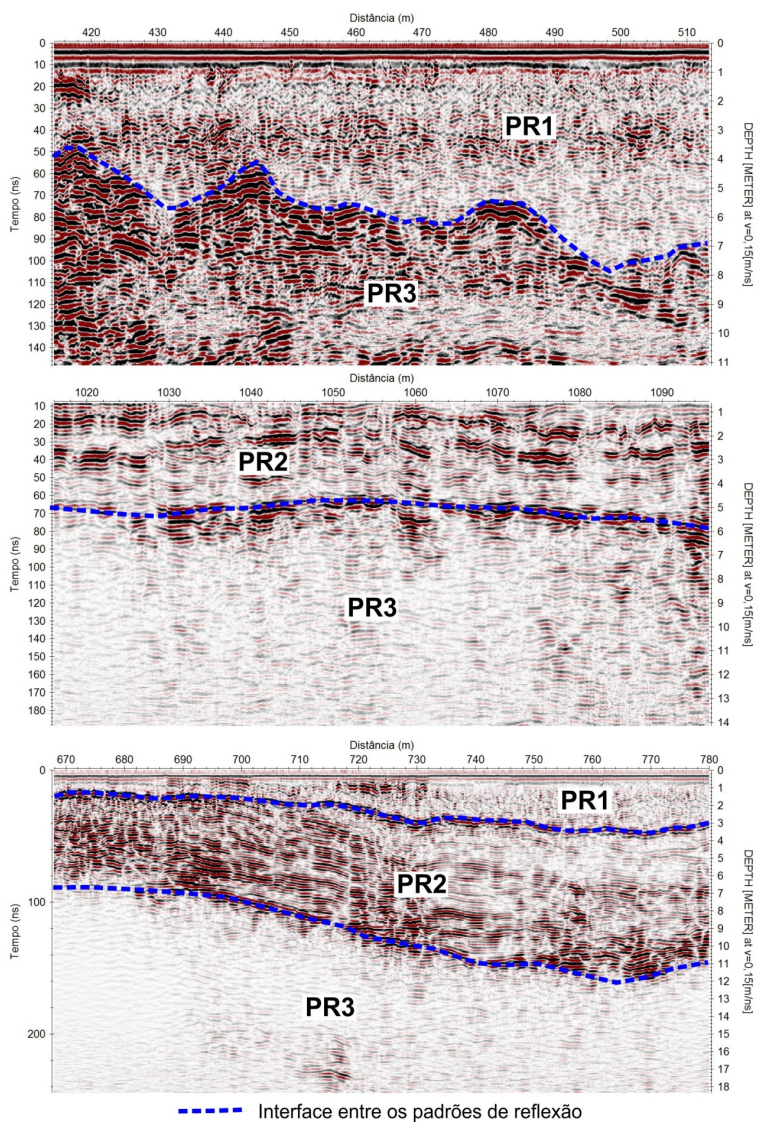

Figura 6 - Seções de GPR evidenciam o padrão de reflexão (PR3) característico do embasamento rochoso. Ele ocorre tanto abaixo de PR1 quanto de PR2.

Apesar de o GPR registrar espessuras de até 6,1 metros de areia maciça na área do Areal Morro Branco, a análise estatística, realizada em 22401 pontos amostrados dos 5 perfis de GPR indica uma espessura média de 3,5 metros, com um desvio padrão de $\pm 0,8$ metros. 
Usando-se uma área aproximada de 8 hectares (200 metros x 400 metros), e uma espessura de 3,5 metros, estima-se uma reserva inferida de $280.000 \mathrm{~m}^{3}$ de areia maciça para o Areal Morro Branco.

A camada de cascalho com matriz arenosa, apesar de atualmente não lavrada, mostra espessuras de até 13,8 metros, o que a torna economicamente viável no futuro. Esta camada registra uma espessura média de 10,3 metros, com um desvio padrão de $\pm 1,5$ metros. Utilizando-se a área atual do Areal, calcula-se uma reserva inferida de $824.000 \mathrm{~m}^{3}$ de cascalho com areia.

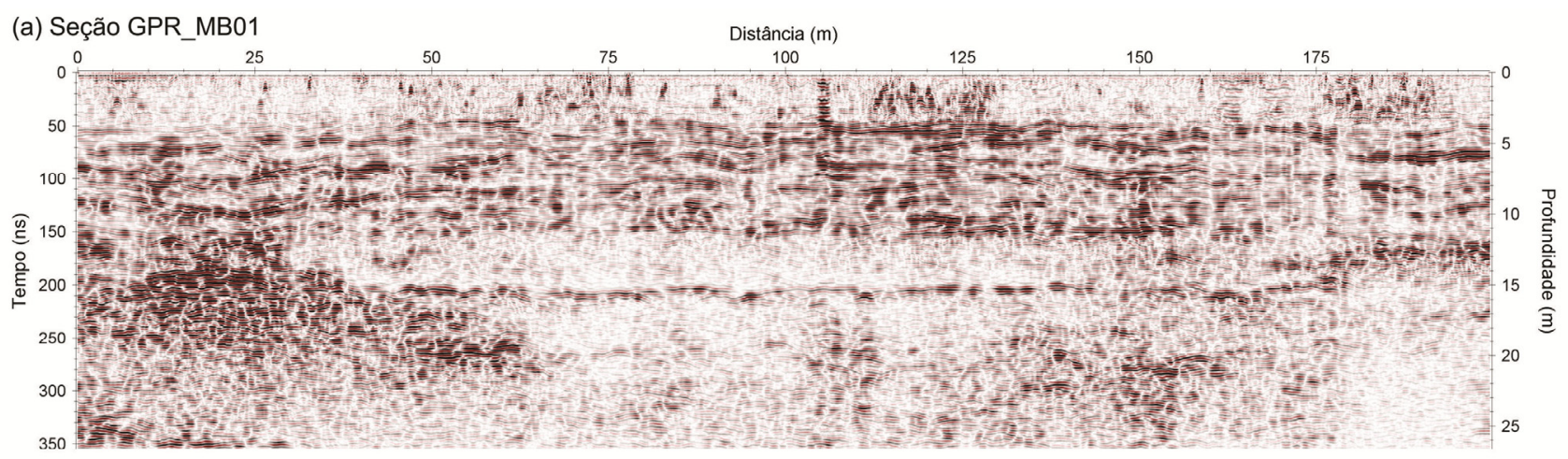

(b) Seção GPR_MB01 com a interpretação sobreposta

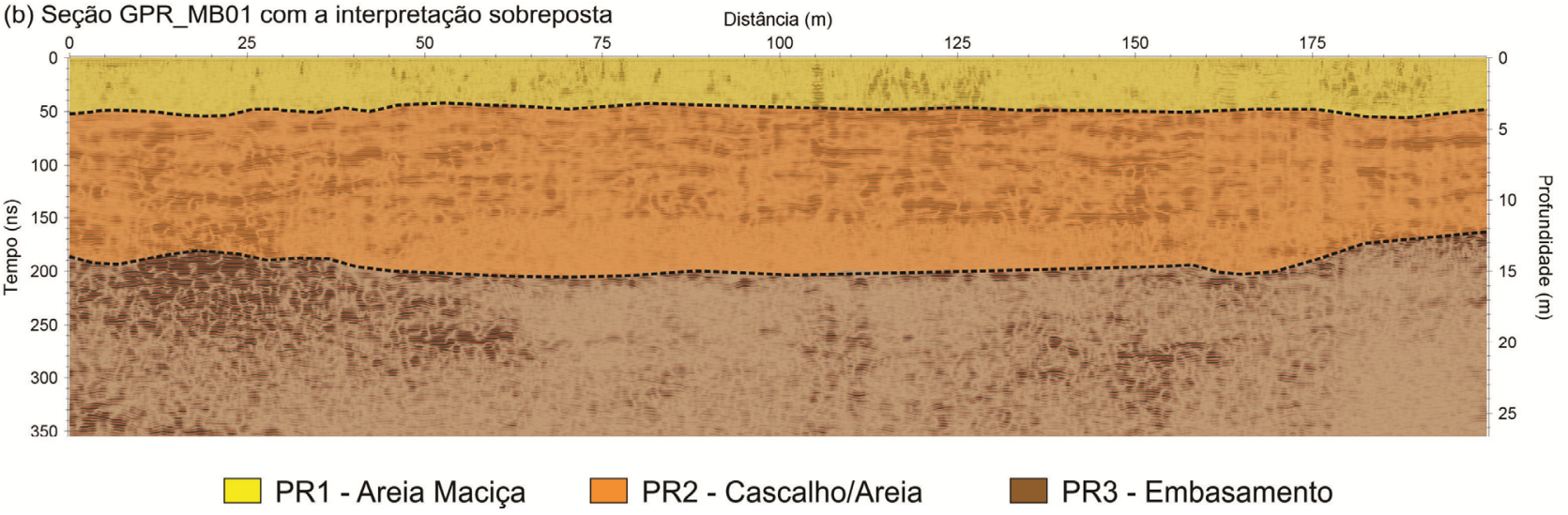

Figura 7 - Seção GPR_MB1 realizada no Areal Morro Branco. (A) Seção sem a interpretação. (B) Seção com a interpretação sobreposta.

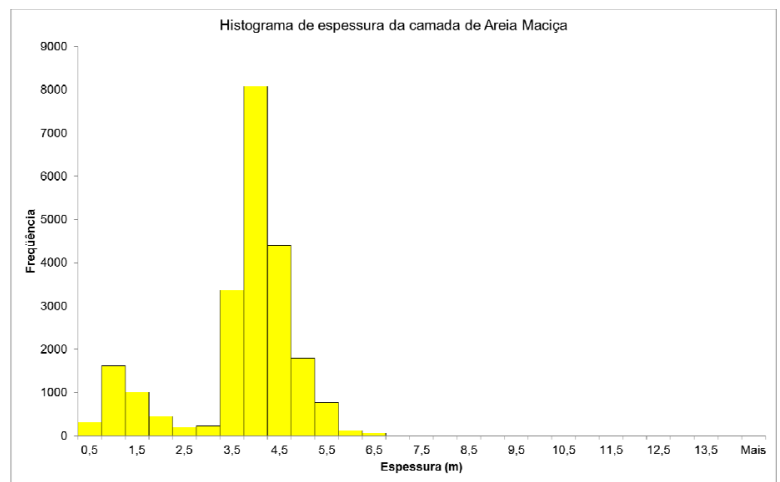

Figura 8 - Histograma de distribuição de frequência das espessuras da camada de areia maciça registradas com o GPR na área do areal Morro Branco.

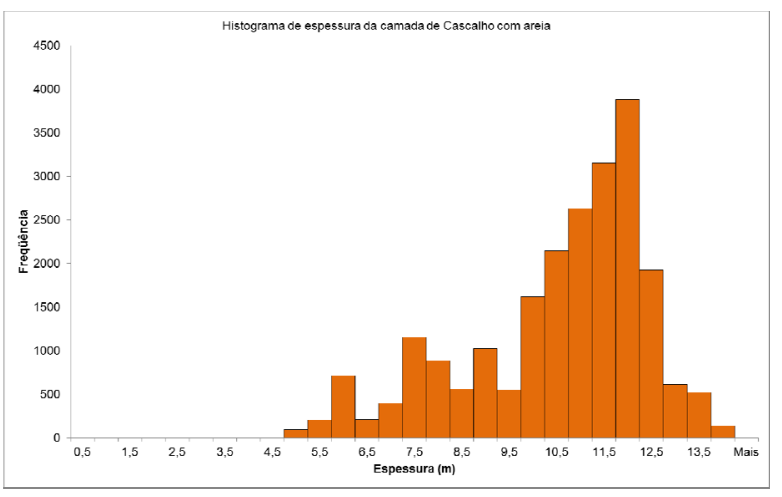

Figura 9 - Histograma de distribuição de frequência das espessuras, da camada de Cascalho com areia, registradas com o GPR na área do areal Morro Branco. 


\section{Conclusões}

As investigações de radar de penetração no solo realizadas na área do areal Morro Branco obtiveram sucesso no imageamento das camadas de areia maciça, de cascalho com areia, e do embasamento rochoso.

Os padrões de reflexão de GPR possibilitaram a individualização destas camadas sedimentares. O padrão de reflexão PR1 (ausência de refletores contínuos e presença de hipérboles de difração) caracteriza a areia maciça. O padrão de reflexão PR2 (refletores contínuos, planos a inclinados, e de alta amplitude) define a presença de camadas de cascalho interdigitando com camadas de areia grossa. O padrão de reflexão PR3 (refletores de alta a baixa amplitude, com geometria de superfícies erosivas) é característico do embasamento rochoso.

O GPR possibilitou estimar o volume de areia no depósito Morro Branco. A análise em campo do tipo de material e a baixa atenuação do sinal do GPR confirmam o alto potencial da areia deste depósito para o uso na construção civil em função da baixa concentração de argila na matriz.

Em função dos resultados obtidos, pode-se afirmar que o radar de penetração de solo é uma ferramenta muito eficiente na determinação dos volumes dos pacotes sedimentares arenosos na região dos paleocanais abandonados dos rios no Amapá.

\section{Agradecimentos}

Os autores agradecem ao Serviço Geológico Brasileiro (CPRM) pelo financiamento de todas as etapas de campo.

\section{Referências Bibliográficas}

BRISTOW, CS; JOL, HM. 2003. Ground Penetrating Radar in Sediments. Geological Society of London, 330P.

SANDMEIER, K.J. 2012. REFLEXW Version 7.0 for Windows $9 \times / 2000 / N T / X P$. Program for the processing of seismic, acoustic or electromagnetic reflection, refraction and transmission data. Manual do Software, 192p. 